\title{
POSITIVE MATRICES AND EIGENVECTORS $\dagger$
}

\author{
by C. R. PUTNAM \\ (Received 19 February, 1962)
}

1. For $i, j=1,2, \ldots$, let $a_{i j}$ be real. A matrix $A=\left(a_{i j}\right)$ will be called positive $(A>0)$ or non-negative ( $A \geqq 0$ ) according as, for all $i$ and $j, a_{i j}>0$ or $a_{i j} \geqq 0$ respectively. Correspondingly, a real vector $x=\left(x_{1}, x_{2}, \ldots\right)$ will be called positive $(x>0)$ or non-negative $(x \geqq 0)$ according as, for all $i, x_{i}>0$ or $x_{i} \geqq 0$. A matrix $A$ is said to be bounded if $\|A x\| \leqq M\|x\|$ holds for some constant $M, 0 \leqq M<\infty$, and all $x$ in the Hilbert space $H$ of real vectors $x=\left(x_{1}, x_{2}, \ldots\right)$ satisfying $\|x\|^{2}=\sum x_{i}^{2}<\infty$. The least such constant $M$ is denoted by $\|A\|$. If $x$ and $y$ belong to $H$, then $(x, y)$ will denote as usual the scalar product $\sum x_{i} y_{i}$. Whether or not $x$ is in $H$, or $A$ is bounded, $y=A x$ will be considered as defined by

$$
y_{i}=\sum_{j} a_{i j} x_{j}
$$

whenever each of the series of (1) is convergent.

When $A$ is bounded, $A^{*}$ will denote its adjoint, $A^{*}=\left(\dot{a_{j i}}\right)$, and $\operatorname{sp} A$ will denote its spectrum, that is, the set of complex numbers $\lambda$ for which $A-\lambda I$ fails to have a bounded (right and left, necessarily unique) inverse. The point spectrum consists of those $\lambda$ in $\operatorname{sp} A$ for which $A x=\lambda x$ holds for some $x \neq 0$ in the Hilbert space $H$.

It is known, as a generalization of the Perron-Frobenius theory $([4],[5],[13])$ for finite matrices, that if $A \geqq 0$, then $\mu=\sup \{|\lambda|: \lambda \in \operatorname{sp} A\}$ also belongs to sp $A$; see, e.g., [2] (cf. pp. $148 \mathrm{ff}.),[8],[14],[15]$. In addition, it is known that under certain additional restrictions on $A$, e.g., that of complete continuity, $\mu$ is in the point spectrum of $A$ and there exists a characteristic vector $x$ in $H$, satisfying $x>0$ or $x \geqq 0$ according as $A>0$ or $A \geqq 0$; see [8], [11], [14]. In case $A>0$, then also $\mu$ is a simple eigenvalue.

If it is assumed only that $A$ is bounded and that $A>0$, then $\mu$ need not be an eigenvalue. (The Hilbert matrix cited below is such an example. Also, any Toeplitz matrix $A=\left(a_{i j}\right)$ given by $a_{i j}=b_{i-j}$, where $\left\{b_{k}\right\}(k=0, \pm 1, \pm 2, \ldots)$ is a sequence of positive numbers for which $A$ is bounded, will do; cf. [7], p. 868.) Thus, in general, $\mu$ need not have an associated eigenvector in Hilbert space. On the other hand, it may happen that there exists a vector $x$ not in Hilbert space for which $A x=\mu x$. This problem has been considered in particular by Kato [9], [10] and Rosenblum [17]. The Hilbert matrix $A=\left((i+j)^{-1}\right)$ satisfies $A>0$ and is bounded; in fact $\mu=\pi$ (see [6], Chapter IX) and, moreover, $\mu$ is not in the point spectrum of $A([12],[18])$. It was shown by Kato [9] in connection with a problem posed by Taussky [19], that $\mu$ does however have a positive eigenvector $x$ not belonging to $H$.

The present paper will consider the problem of the existence of vectors $x>0$, not necessarily in $H$, associated with certain bounded $A>0$, for which

$$
A x \leqq \mu x ; \quad \mu=\sup \{|\lambda|: \lambda \in \operatorname{sp} A\} .
$$

In Theorem 1 it will be shown that the inequality of (2), when $x$ is in $H$, implies equality under $\uparrow$ This research was supported by the U.S. Air Force Office of Scientific Research. 
certain circumstances, while in Theorem 2 it will be shown that (2) must always hold for some vector $x$, not necessarily in Hilbert space. This last result will be combined with a theorem of Kato to yield Theorem 3, giving a necessary and sufficient condition for the boundedness of a symmetric positive matrix.

\section{There will be proved the following}

Theorem 1. Let $A$ be bounded and satisfy $A>0$. Suppose that (2) holds for some $x>0$ of the Hilbert space $H$. Suppose in addition that either $\mu$ belongs to the point spectrum of $A^{*}$, or only that there exists some $v$ satisfying $|v|=\mu$ and belonging to the point spectrum of $A^{*}$. Then necessarily equality must hold in (2), so that

$$
A x=\mu x \text {. }
$$

Furthermore, $\mu$ must then be a simple eigenvalue of both $A$ and $A^{*}$, and both have positive eigenvectors (each unique except for a positive multiple).

It can be remarked that in case $A$ is completely continuous, then $\mu$ belongs to the point spectrum of both $A$ and $A^{*}$, so that, in particular, the hypothesis of the theorem concerning $A^{*}$ is fulfilled.

Proof of Theorem 1. Let $A^{*} y=v y$, where $|v|=\mu$ for some $y=\left(y_{1}, y_{2}, \ldots\right) \neq 0$ of the Hilbert space $H$. If $|y|$ is defined by $|y|=\left(\left|y_{1}\right|,\left|y_{2}\right|, \ldots\right)$, it is clear that $|y|$ is also in $H$ and that

Hence, by (2),

$$
A^{*}|y| \geqq \mu|y| \text {. }
$$

$$
(\mu x,|y|) \geqq(A x,|y|)=\left(x, A^{*}|y|\right) \geqq(x, \mu|y|) .
$$

Thus the inequalities of (5) become equalities. In particular the last yields

$$
\left(x, A^{*}|y|-\mu|y|\right)=0
$$

and hence, by (4) and the fact that $x>0, A^{*}|y|=\mu|y|$. But $A^{*}>0$, and this implies that $|y|>0$. The first relation of (5) now becomes $(A x-\mu x,|y|)=0$, which yields (3), as a consequence of (2) and $|y|>0$. The last assertion of the theorem can be proved as in [14] (cf. p. 590).

The argument used above is similar to that used in $[16, \mathrm{pp} .78-80,82]$ for integral equations.

3. In this section it will be shown that, for every bounded $A>0$, there exists some positive vector $x$, not necessarily in Hilbert space, for which (2) holds. Whether there exists a relation corresponding to (3) under conditions similar to those of Theorem 1 will remain undecided however. There will be proved the following

THEOREM 2. Let $A$ be bounded and satisfy $A>0$. Then there exists a vector $x>0$, not necessarily in Hilbert space, for which (2) holds.

Proof of Theorem 2. Let $\lambda$ be real and satisfy $\lambda>\mu$. Since the resolvent $R(\lambda)=(A-\lambda I)^{-1}$ satisfies $(A-\lambda I) R(\lambda)=I$, it follows that

$$
A R(\lambda) y=\lambda R(\lambda) y+y
$$

where $y=\left(y_{k}\right)$ is any vector of Hilbert space. If $R(\lambda)=\left(r_{i j}(\lambda)\right)$ and if $z=R(\lambda) y$, so that 


$$
z_{k}=\sum_{m} r_{k m}(\lambda) y_{m} \quad(k=1,2, \ldots)
$$

then, by (6),

$$
(A z)_{k}=\lambda z_{k}+y_{k}
$$

Since $\lambda>\mu(\mu$ being the spectral radius of $A), R(\lambda)$ is given by $R(\lambda)=-\sum_{n=0}^{\infty} A^{n} \lambda^{-n-1}<0$. Choose $\lambda_{1}>\lambda_{2}>\ldots \rightarrow \mu+0$ and let, for each $n=1,2, \ldots, y^{(n)}=\left(y_{k}^{(n)}\right)$ be defined by

$$
y_{k}^{(n)}=\left(r_{1 k}\left(\lambda_{n}\right)\right)^{-1} \text { or } 0 \text { according as } k=n \text { or } k \neq n \text {. }
$$

Then, if $z^{(n)}=\left(z_{k}^{(n)}\right)$, one obtains from (7) the relation

$$
z_{k}^{(n)}=\sum_{m} r_{k m}\left(\lambda_{n}\right) y_{m}^{(n)}=r_{k n}\left(\lambda_{n}\right) / r_{1 n}\left(\lambda_{n}\right)>0
$$

and, in particular,

$$
z_{1}^{(n)}=1 \text { for } n=1,2, \ldots
$$

Since $y_{k}^{(n)} \rightarrow 0$ as $n \rightarrow \infty$ for each fixed $k=1,2, \ldots$, it follows that $\left\{\lambda z_{1}^{(n)}+y_{1}^{(n)}\right\}$ is a bounded sequence of numbers. According to (8), for $k=1$, this last expression is equal to $\left\{\sum a_{1 m} z_{m}^{(n)}\right\}$; hence, since $A>0$ and $z_{k}^{(n)}>0,\left\{z_{k}^{(n)}\right\}$ is a bounded sequence of numbers for each fixed $k=1,2, \ldots$. By the diagonal selection process there exists a sequence $\mu_{1}>\mu_{2}>\ldots$ $\rightarrow \mu+0$ for which

$$
x_{k}=\lim _{n \rightarrow \infty} Z_{k}^{(n)} \quad \text { exists for each } k=1,2, \ldots,
$$

where $Z_{k}^{(n)}$ is defined by $(10)$ with $Z_{k}^{(n)}=z_{k}^{(n)}$ and $\lambda_{n}$ replaced by $\mu_{n}$. Clearly $x=\left(x_{1}, x_{2}, \ldots\right) \geqq 0$. In addition, it follows from (8) that

$$
\mu x_{k}=\lim _{n \rightarrow \infty}\left(\sum_{m} a_{k m} Z_{m}^{(n)}\right) \geqq(A x)_{k}, \quad \text { for } k=1,2, \ldots .
$$

Hence (2) holds and so $x>0$ by virtue of (11) and $A>0$. This completes the proof of Theorem 2 .

4. As a consequence of a result of Kato $[10$, p. 576] and Theorem 2 of the present paper there will be proved

THEOREM 3. Let $A=\left(a_{i j}\right)$ be any symmetric positive matrix $\left(0<a_{i j}=a_{j i}\right)$, not assumed to be bounded. Then a necessary and sufficient condition that $A$ be bounded is that there exist some real constant $v$ and a vector $x>0$, not necessarily in Hilbert space, for which

$$
A x \leqq v x
$$

Proof of Theorem 3. The sufficiency follows from the result of Kato mentioned above, even if the hypothesis $A>0$ is weakened to $A \geqq 0$. In fact, it is shown there that

$$
\|A\| \leqq v .
$$


The necessity follows from Theorem 2 above. In fact, if $A$ is bounded and positive, even if $A$ is not symmetric, then (2) holds for some $x>0$. This completes the proof of Theorem 3 .

Incidentally, it is clear that relation (2) for some $x>0$ implies (14) for the same $x$ and all real $v>\mu$. Since $\|A\|=\mu$, it follows from (15) that (14) then holds for some fixed $x>0$ and for all $\nu \geqq \mu$, but that (14) does not hold for any $x>0$ if $v<\mu$.

\section{REFERENCES}

1. F. F. Bonsall, Endomorphisms of partially ordered vector spaces, J. London Math. Soc. 30 (1955), 133-144.

2. F. F. Bonsall, Endomorphisms of a partially ordered vector space without order unit, ibid. 30 (1955), 144-153.

3. F. F. Bonsall, Linear operators in complete positive cones, Proc. London Math. Soc., Ser. 3, 8 (1958), 53-75.

4. G. Frobenius, Ueber Matrizen aus positiven Elementen, Sitzungsberichte Preuss. Akad. Wiss. (Berlin, 1908), 471-476; (1909), 514-518.

5. G. Frobenius, Ueber Matrizen aus nicht negativen Elementen, ibid. (1912), 456-477.

6. G. H. Hardy, J. E. Littlewood and G. Polya, Inequalities (Cambridge, 1952).

7. P. Hartman and A. Wintner, The spectra of Toeplitz matrices, Amer.J. Math. 76 (1954), 867882.

8. S. Karlin, Positive operators, J. Math. and Mech. 8 (1959), 907-937.

9. T. Kato, On the Hilbert matrix, Proc. Amer. Math. Soc. 8 (1957), 73-81.

10. T. Kato, On positive eigenvectors of positive infinite matrices, Comm. Pure and Applied Math. 11 (1958), 573-586.

11. M. G. Krein and M. A. Rutman, Linear operators leaving invariant a cone in a Banach space Uspehi Mat. Nauk (N. S.) 3, No. 1 (23) (1948), 3-95, Amer. Math. Soc. Trans. No. 26.

12. W. Magnus, On the spectrum of Hilbert's matrix, Amer. J. Math. 72 (1950), 699-704.

13. O. Perron, Zur Theories der Matrices, Math. Annalen 64 (1907), 248-263.

14. C. R. Putnam, On bounded matrices with non-negative elements, Canadian J. Math. 10 (1958), 587-591.

15. C. R. Putnam, A note on non-negative matrices, ibid. 13 (1961), 59-62.

16. M. A. Rutman, Sur les opérateurs totalement continus linéaires laissant invariant un certain cone, Mat. Sbornik (N. S.) 8, no. 1 (50) (1940), 77-96.

17. M. Rosenblum, On the Hilbert matrix, I, Proc. Amer. Math. Soc. 9 (1958), 137-140.

18. O. Taussky, A remark concerning the characteristic roots of the finite segments of the Hilbert matrix, Quart. J. Math., Oxford Ser., vol. 20 (1949), 80-83.

19. O. Taussky, Research Problem 60-3-12, Bull. Amer. Math. Soc.

PuRdue UNIVERSITY 